\title{
Bayesian Network with CSA to optimize the selection of QoS- aware Web service
}

\author{
Saravanan Chandrasekaran \\ Research scholar Department of computer science and Engineering Annamalai University Tamil Nadu,India \\ availableathere@gmail.com
}

Vijay Bhanu Srinivasan

Assistant Professor, Department of computer science and Engineering Annamalai University

Tamil Nadu, India

vbhanu22@yahoo.in

Latha Parthiban

Assistant Professor,Department of Computer Science Pondicherry University

Pondicherry, India

lathaparthiban@yahoo.com

\begin{abstract}
The Quality of Service (QoS) is a crucial factor for selecting a web service. It is one among the major multi objective optimization problem so the selection of QoS aware web service is always crucial. The predicted values of the QoS are likely to fluctuate due to the unpredictable network connection and user environment. In this paper, the Bayesian Network (BN) with Cuckoo Search Algorithm (CSA) is proposed for optimizing the selection of QoS-aware web service. The proposed BN-CSA model approach, takes into account the response time, reliability and accuracy for the selection of QoS-aware web service. Data for training purpose is developed and to calculate the best trained data by utilizing the CSA, is a optimization algorithm which is based on meta Heuristic and it is suitable for solving optimization problems. The parameters of the cuckoo search are kept constant and the steps of CSA are selection, creation and updating. Problems with multi-objective optimization are solved with the advantage of fitness function. The proposed optimization process is built in JAVA platform and its performance is examined and compared with other existing techniques, the proposed BN-CSA outperforms with fast convergence and less execution time to find the most optimized fitness value.
\end{abstract}

Keywords- Quality of Service; Web Service; Cuckoo Search Algorithm; Bayesian Network.

\section{INTRODUCTION}

In the recent years, Web services technology has been emerging and is the most popular techniques for distributed software development [1]. Some web services like UDDI, BPEL, SOAP, WSDL, WSFL, XLANG having merits with business processes building because of its standard, slackly attached, returnable, flexible, and effective nature [2]. Based on the quality of the web services been employed, does the quality of service-oriented systems depends. Web Services are self-independent application that shows modular and also distributed concepts. Web service description is provided in the WSDL document and it can be accessed from the internet using SOAP protocol [3]. For Enterprises information integration Service Oriented Architecture (SOA) is one of the core technology which is fundamentally a throng of web services which are interact to one another in that the network [4]. Web services composition consists of multiple service nodes, in which all the nodes resemble to a service from the pool of a community of services (i.e., a group of functionally similar web services). Therefore, the number of composition plans increases with the increasing number of web services per community [5]. With the increasing popularity of the development of SOA, measuring the quality of services becomes an imperative concern for service consumers and providers [6]. Web services are selected based on their functional properties or nonfunctional, Quality of Service (QoS), properties [7, 8].

For a particular assigned service request, service arrangement hunt for a service composition solution which meets nonfunctional requirements which is not limited to functional requirements. Where it is known as Quality of Service -aware service composition, the aim behind this QoS-aware web service configuration is to ascertain the best composition of web services which complies with the functional necessities and encounters the end-to-end QoS requirements [9]. Numerous solicitations necessitate the need of web services to choose and synchronize with other web services in particularly in e-commerce activities, semantic web and peer-to-peer networks. Examples of current systems involved in such activities include Amazon, eBay, Expedia, etc. This organization comprises treasured possessions such as period, cash, and hardware practice [10]. The definition of Quality of Service based web service is on the basis of diverse Service Level Agreement (SLA) open among patrons and the service providers. The composite service the QoS is to swung in the internet-centered situation due to the worth of tangible services, which owing to many aspects which are exposed to modification. The user request can be associated with 
some end-to-end QoS requirements like service price, response time and availability respectively [11, 12]. The service composer has to ensure that the aggregated QoS values of the selected services match the user requirements, and obviously, users hope to subscribe the combination of services with optimal QoS performance, while fulfilling their Quality of Service constraints $[13,14]$. The problem of such kind can be coined as the MultiConstrained Optimal Path (MCOP) Problem, which is known to be NP hard.To achieve shorter response time, a network service requires much faster composition of algorithms due to the real-time requirements of various network protocols [15].

QoS-aware web services selection has been proven NP-hard problem [16]. Many factors lead to this high complexity such as (i) the users set different constrains for selection and compositions, (ii) QoS parameters of a web service differ than what is claimed by its provider, (iii) Web Services like functionalities available in many numbers and (iv) within this huge search space, compositions may be built by many ways [17]. The last two factors increase time complexity of the web services selection. Early research solves web service selections as linear programming problems [18]. Consistent QoS Provisioning by the network for eace and every service is a main goal behind Quality of service Optimization and adaptation. The Optimal QoS Selection of parameters are represented by variable application requirements of the network. To achieve the user requested goals, a set of system-specific parameters are included in the fitness function. In the process of creating optimal working conditions in web service network, detailed and clear evaluation of parameters obtained through simulation and measurements is crucial play and also very important role in the above process. In line with the fulfillment of constrains in both functional and non-functional suitable services has to be chosen for service composition [19]. An efficient BNCSA is proposed in this paper to optimize the selection of Quality of Service aware web services. The rest of this paper is organized as follows. Section II discusses the Literature Review. The Proposed model is presented in Section III. Results are discussed in Section IV, and section V discusses about the conclusion.

\section{LITERATURE REVIEW}

Numbers of research works are previously proposed to optimize the selection of QoS aware web service. In [20] the resulting composition of semantic I/O based with optimal E-to-E Quality of Service presented as a hybrid approach in the automatic composition of Web Services which generates the above has four main steps: 1) For a request a graph has to be generated; 2) In a single objective Quality of Service minimization task an optimal composition has to be computed; 3) In the process of minimization of search space through the route of finding and dominated services multi-step optimization is employed; and 4) To extract the optimal Quality of Service with the least figure of services a hybrid local-global search is used. The following points indicates that An widespread justification of datasets of the Web Service Challenge 2009-2010 and arbitrarily produced datasets: 1) the arrangement of native and universal optimization was a categorized and commanding method to getting the things an prime masterpieces in dissimilar circumstances; and 2) the mixture approach executes superior in comparisons with the state-of-the-art, attaining results with less services and prime Quality of Service.

Ref. [21] have examined Service composition. This analysis offers a value of personalized and springy combination of service functionalities the non-functional desires of the user is designated as a work flow in QoS optimization algorithm. Previously implemented tactics miscarry to reach an enough consistency by way of swelling figure of services in a work-flow. Moreover, to deal with service failures expensive ad-hoc re-planning becomes unavoidable. Out of desk plan such as potential cost is not included during plan pottering and also hided from decision maker during approval is the main problems in sequential application of planning and re-planning. The intention of this paper is to overcome the problem and computation of a Quality of Service elevated range of service clusters which comprises a enough quantity of standby services for all services engaged. In this paper we included the total repair cost on the planning itself to make easy for the decision make.

A innovative methodology centered on a Harmony Search algorithm which speaks those three dimensions instantaneously over a fitness function, to hand-picked the ideal or near-ideal result in semantic web service alignment [22]. The contribution is to typical the search space as forecasting graph organization in which at any given user request is encoded as many as possible solutions which is to be called as Planning Graph Structure. The web service challenges 2009 conducted a series of tests on the algorithms of Improved Harmony Search along with the Global Best Harmony Search from that the following conclusions arrived (1) the work intended is competent and operative to get the ideal or near-ideal configuration in assorted circumstances; and (2) fitness and execution time improvement is witnessed together options IHS and GHS algorithms.

As the outcome of composite services instance is to enlarge the values of Quality of Service put together as a focus for Quality of Service aware Optimal Service composition in the meantime assembling of user-stated universal Quality of Service limitations Non-consideration of multi-abstract service while doing one abstract service in the composite service during the candidate service is the process of traditional methods [23]. have proposed the concept of generalized component services (GCSs), to overcome the shortcoming, which was clear in a semantic fashion, in the route to attain better solutions the selection scope is expanded. A QoS-aware multigranularity service conformation prototypical was conveyed and how to recognize all the GCSs to a particular 
composite service was expounded. In line with the optimization of the service instance of resulting composites is proposed by a back-tracking based algorithms in combination with extended genetic algorithm.

With the advent of cloud manufacturing (CMfg) more and more services in CMfg platforms may offer the similar functionality then again fluctuate in performances. In the process of matching the manufacturing cloud with complicated task requirements is very needful to become in composite CMfg service optimal selection (CCSOS). [24] have anticipated the hybrid artificial bee colony (HABC) for such CCSOS problems, in which the likelihood of typical of Archimedean copula estimation of distribution algorithm (ACEDA) and the chaos operators of global best guided artificial bee colony are employed to make the descendants entities by way of the deliberation of Quality of Service and CMfg atmosphere. To assess the recital of the wished-for HABC, different-scale CCSOS complications were adopted. When employing web services in places like unreliable environment the risk of failure is witnessed. To get assured for reliable execution in the transaction mechanism is wanted by composite web services (CWS) while execution. Quality of service and transactions are considered separately by available service selection methodologies and wont includes transaction properties might degrade the Quality of Service like Execution time of CWS. [25] have spoken the significance of the impact of Quality of Service in the continuing chain of service choice by Quality of Service aware and transitional aware selection approach is elaborated. (1) In the transactional CWS, calculations of Process duration are examined. (2) To achieve universal optimization service selection, the following parameters of CWS is included successful process running time, Behaviors of transactions, cost, Process duration have taken into account in genetic algorithm based approach. Finally, (3) Usefulness and competence of the method is established with the investigational test results.

Performing effective ranking prediction for cloud services can help customers make prompt decisions when they were confronted by a large number of choices. The method can also enhance web service user satisfaction levels. Improving ranking prediction of QoS-based services continues to be an active topic of research in cloud service recommendation. Most service recommendation algorithms focus on prediction accuracy, ignoring diversity, which also may be an important consideration. [26] analyzed service recommendation as a multiobjective optimization problem, and give two modified ranking prediction and recommendation algorithms that simultaneously consider accuracy and diversity. Existing algorithm recommendations can be made much more diverse by adjusting weights on service origin and substantially reducing the risk of inappropriate recommendations. Our experiments show that the algorithms can propose yield greater diversity without greatly sacrificing prediction accuracy.As the numeral of web services that offer analogous functionality rises, In the process of grading and electing of accurate web services Quality of Services becomes vital. The difficulties of Web Services selection for Web Service composition comprises three core aspects which includes the complex services process and numerous performance necessities for samples an end-to-end delay, facility price and consistency of a multifarious service. The unintentional demerits of QoS Prediction approaches are: In a exact circumstance constructing superior depiction with the precision of estimation in a particular period is owned by single models only. All activities oriented towards structure, grid, carriage, and middle ware support for Quality of Service Therefore, QoS-aware web service alignment can be seen as a NP-hard unruly and determined by numerous procedures comprising arithmetical exhibiting, computational intelligence techniques and operational research,. Traditionally, most intelligent techniques like Fuzzy system, Particle Swarm Optimization (PSO), Ant Colony Optimization (ACO), Genetic Algorithm (GA), and self-organizing migrating algorithm (SOMA) are performed to optimize the Selection of QoS-aware Web Service. And a good forecast exemplary must fulfill all the definite circumstances. Therefore various forecast methodologies can be pooled to expand prediction exactness, effectiveness and expand the application scope. In the paper, an effective optimization algorithm is required for selection of QoS aware Web Service. In the paper, an effective optimization algorithm is required for choice of Quality of Service aware Web Service. There are many parameters of quality on which service discovery is dependent on like reliability, availability, throughput and accuracy, performance, etc. The technique finds out fitness function that is having high robustness and removes the error which would not affect the system.

\section{PROPOSED MODEL}

Most of the prediction system chooses the Web services based on the QoS factor. Web services with similar functionality are grouped into communities for better exposure. Those similar web services are distinguished by their QoS parameters during service selection. The calculations and estimation prototypes of QoS have been analyzed in countless conditions, principally for computer networks and multimedia applications [27].

\section{A. QoS Attributes}

To describe the nonfunctional behavior of Web Services Quality of Service is chosen and divergent way in the route of picking and resembling services from functionally the same one. The entire service provider defines the Quality of Service model before distributing Quality of service aware delivery service. The following parameters have been considered in the integral part of web services such as consistency, reaction period, readiness and price as well QoS features also applied apart from those things 0 and 1 is the range of Quality of Service factors. Each Service provider held their value of all Quality of Service. The 
maxima and minima is set by the consumer to the service provider namely, for positive attributes the upper bound is suggested such as consistency and readiness and the lower bound is suggested for negative attributes like process price and process time. Different QoS parameters describe the quality in different ways. The QoS attributes need to be normalized and uniformly scaled using (1) for negative attributes and (2) for positive attributes as illustrated below,

$$
\begin{aligned}
& D_{i}\left(s_{k}\right)=\left\{\begin{array}{c}
\frac{D_{i}^{\text {max }}-d_{i}\left(s_{k}\right)}{D_{i}^{\text {max }}-D_{i}^{\text {min }}} ; D_{i}^{\text {max }}-D_{i}^{\text {min }} \neq 0 \\
1 ; \quad D_{i}^{\text {max }}-D_{i}^{\text {min }}=1
\end{array}\right. \\
& D_{i}\left(s_{k}\right)=\left\{\begin{array}{cl}
\frac{d_{i}\left(s_{k}\right)-D_{i}^{\max }}{D_{i}^{\text {max }}-D_{i}^{\min }} ; & D_{i}^{\max }-D_{i}^{\min } \neq 0 \\
1 ; & D_{i}^{\max }-D_{i}^{\min }=1
\end{array}\right.
\end{aligned}
$$

Where,

i, represents the number of QoS attributes.

$s_{k}$, is defined as the quality of web services.

$D_{i}$ is denoted as the QoS parameter of web service

$D_{i}^{\max }$ and $D_{i}^{\min }$ are expressed the maximum and minimum values provided by the user for a particular QoS attribute.

In the best possible way, QoS In all the Service-Level Agreements (SLAs) by service suppliers values of services straight away specified. Most of the Quality of Service provisions might turn out to be inexact due to the succeeding appearances:

-Vary the time: The frequently fluctuating working atmosphere, such as changing network delays throughout the Internet and energetic facility capacities, marks numerous Quality of Service attributes (e.g., reaction period and throughput) distributed to consumers swing extensively over time.

-User-identified: Each and every user have different Quality of Service Values for similar Quality of Service or even for the same QoS because of the Wide spared of Georgic area.

These features mark procurement truthful Quality of Service evidence for runtime service employment a demanding function. These papers consider the QoS attributes namely, reaction period, trustworthiness, readiness, throughput and accurateness. All QoS attribute linked with its analogous depiction is analyzed in below. Then the overall unbiased role can be framed as the following as in (3).

$$
O b(F)=1 / n \sum_{i=1}^{n}\left(D_{i} \times W_{i}\right)
$$

Where, $D_{i} \times W_{i}$ represents the interpretationuserpreferencesintermsofweightsforeachattributeand represents the number of QoS attributes involved. In our work, the problem of Quality of Service forecast has been verbalized to leverage historical Quality of Service data witnessed from diverse consumers to precisely evaluate Quality of Service standards of candidate services, while eliminating the need for additional service invocations from the intended users. In order to optimize the selection of QoS-aware web service, the BN-CSA is proposed and evaluated. The performance of proposed method is explained in underneath section.

\section{B. Prediction of QoS}

In this work, we mainly concentrate on Quality of Service miscellany and forecast of web service. The structural view of proposed method is illustrated in Fig. 1. Here, BN with Cuckoo Search Algorithm (CSA)is proposed for optimizing web service selection. The performance of proposed algorithm consists of few parameters and fast convergence speed. And the execution time performance of web services selection to realize the concentrated fitness value in terms of combined Quality of Service is concern till then with the continues increasing number of available web services for a task for practical distributed applications. Moreover, the performance of the $\mathrm{BN}$ is improved by using the CSA. Initially, likenesses amongst the active user and other users in the training data are calculated using the proposed method. In addition that the QoS attributes such as response time, availability, throughput, reliability etc. are given as the inputs of the proposed method. Here, the proposed cuckoo search algorithm is performed to find the best trained dataset values. Subsequently, the trained datasets are formed and are given as the input to the BN. The proposed $\mathrm{BN}$ is used to minimize the error function and optimize the selection of QoS- aware web service. After predicting the QoS values, the web services are ranked according to the QoS values and delivered to the user. 


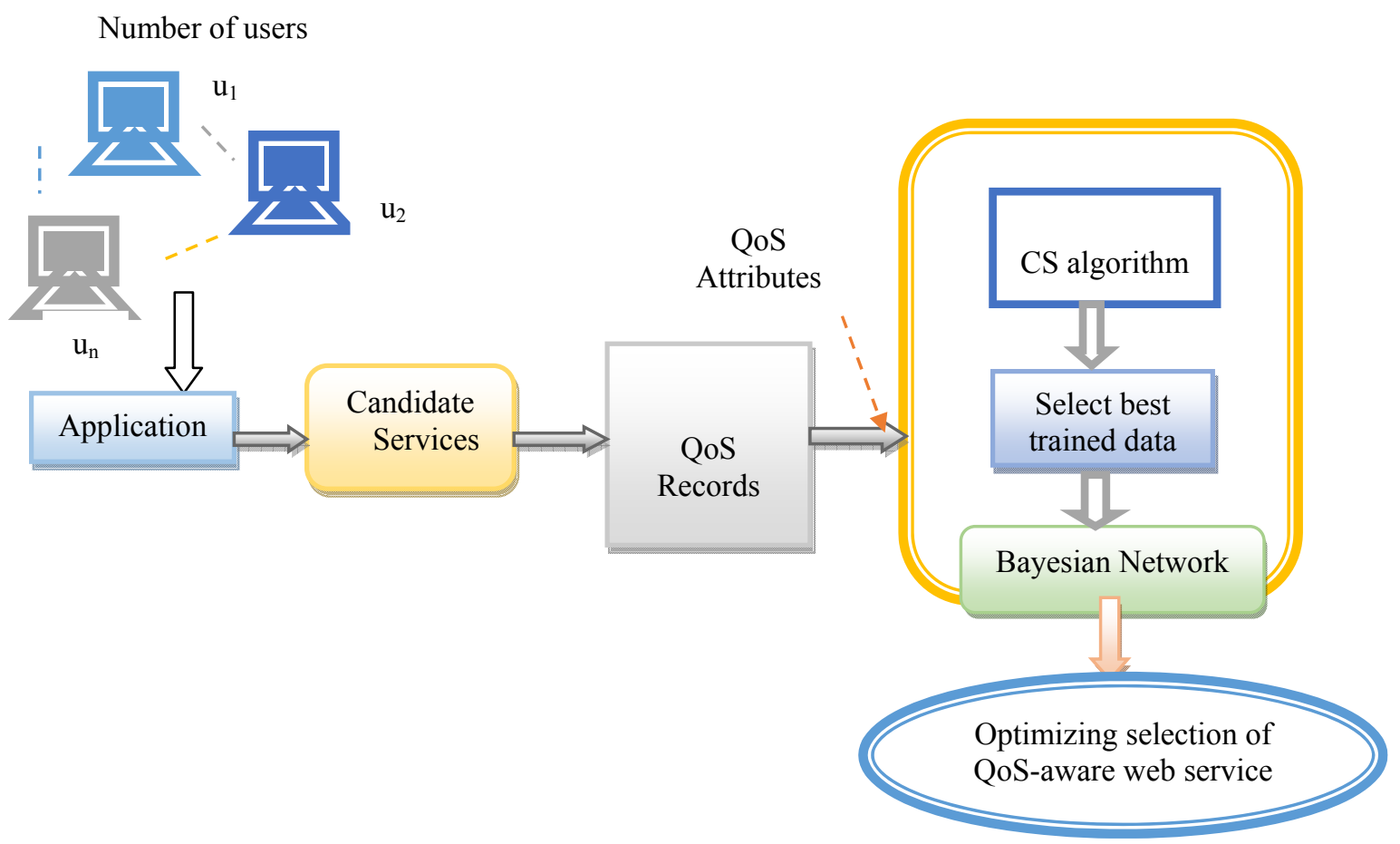

Fig. 1. Prediction of QoS using BN-CSA

\section{Bayesian Network to Optimize the Prediction of QoS value with aid of CSA}

Bayesian networks $(\mathrm{BN})$, is one of the methodology with complete variable and their relationship. It is a graphical representation model. In a general form, structure of BN is a focused acyclic graph (DAG) model which symbolizes conditional independencies amongst a set of variables. It consists of two components: (1) Graphical structure of a network which is a directed acyclic graph with the nodes of variables and arcs of relations [28]. (2) Probability of conditional table which has straight linked with each and every node of the model graph. Structure estimation is done by machine learning language and the probability of conditional table from the training data. Each and every node in the plot is belongs to a discrete random variable of that domain. In one of the graph of such kind an edge, $A \leftarrow B$, on the graph, explains a parent-child relationship in that $A$ is the denoted as child and $B$ is considered as parent. All parents of $A$ belong to the parent set of $A$ which is represented by $\Pi_{A}$. Each and every node in a graph has a conditional probability table (CPT) representing the probability of the corresponding possible state of the node given each realizable condition of its parents. If any one of the node contains no parent, then the table provides very narrow probabilities of that node. Based on the Bayesian probability conclusions, the conditional probability table (CPT) can be calculated from the statistical data and cultivate into the structure of the network [29]. The final probability value will be used through setting up the threshold of confidence as the representation for the cataloging verdict. The Bayesian formula can be mathematically expressed as below in (4)

$$
p\left(h_{j} / V\right)={ }^{p\left(V / h_{j}\right) \cdot p\left(h_{j}\right)} / \sum_{i=1}^{n} p\left(V / h_{i}\right) \cdot p\left(h_{i}\right)
$$

for $\mathrm{j}=1,2,3, \ldots, \mathrm{n}$.

In accordance with the basic statistical theory, for example the Chain Rule and independency relation derived from the network structure, the joint probability of can be calculated by the production of local distributions with its parent node is expressed in (5) as,

$$
p(V)=\prod_{i=1}^{n} p\left(V_{i} \mid \text { parent }\left(V_{i}\right)\right)
$$

Where, $V$ is denoted as the set of variable values i.e. , $V=\left\{V_{1}, V_{2}, \ldots . V_{n}\right\}, h$ is termed as hypothesis.

$h$ denotes the previous probability and $p(h \mid V)$ is known as posteriori probability of $\mathrm{h}$ denoted by $V$. If $V_{i}$ has no origin nodes, then origin of $\left(V_{i}\right)$ is equal to $p\left(h \mid V_{i}\right)$.

In BN structure, the input of best trained data set value is evaluated from the CSA. The CSA generates the best training dataset depends on the input of the Quality of Service features, the inputs are the response time, 
reliability and some other parameters. Moreover the evaluation of best trained data from the CSA is described in the underneath section.

\section{1) To select the best trained data by using CS algorithm}

Cuckoo Search (CS) is a new meta-heuristic optimization algorithm developed by Yang and Deb. For solving the optimization problems it is implemented on the basis of fascinating reproduction character like as household parasitism of group of some cuckoo bird and the characteristics of gathering flights. Cuckoo is a community based search algorithm and it has a group of dens or eggs. The C-S algorithm implemented into the engineering optimization tasks and results in its unavoidable improvements, in the host species nest's cuckoo bird lays her eggs. In the assumption of the eggs are its own by the host, it takes care of it. While the host found that an egg is not its own, it may either demolish the egg or the nest and then construct a new nest at a different location. In the existing solution by varying certain characteristics, new solutions are generated which in turn increase the quality of solutions. A cuckoo egg appears a new results while each and every host cuckoo's egg in a den mentions an outcome. The main purpose is to substitute a subdue outcome in the dens with the new and feasible results. The C-S algorithm will be tender down by three romanticized suppositions, which are:

1) Each and every one of the cuckoo bird deposits one egg at a time, and deposits its egg in indiscriminately selected.

2) The best den with superior grade of eggs or results would convey across to the succeeding generations and

3 ) the den available in number is fixed where the egg deposited by a cuckoo bird is located by the host bird with a measured fraction probability, $p_{a} \in[0,1]$.

According to this paper, the CS algorithm is used for improving the performance of BN. The CS algorithm is generates the training dataset based on the input of the system, the inputs taken from the QoS parameters. Here, the nests are randomly initiated and the cuckoos are generated new solutions using Levy flight function. The fitness function is considered as the minimization of error value. Then evaluate the fitness function and best trained dataset solutions are saved. The optimization is takes place under the following step by step procedure.

\section{Condition 1: Describe the Initialization Process}

In this occurrence, the host bird can besides throwing the egg off or simply desert in the den and raise a totally new den in a new arena

Step 1: Randomized Initialization

Initially, the QoS attributes are randomly generated. The initialized system parameters considered as a function which denoted as $X_{n}$. Initialize a population of $\mathrm{n}$ number of cuckoos at host nest $X^{n}$ that is $(n=1,2,3, .$.$) and the cuckoo's parameters such as QoS data point and its length is randomly described as,$

$$
X_{\min }^{n} \leq X^{n} \leq X_{\max }^{n}
$$

Step 2: Set the iteration count value

Then generate the random population of the $\mathrm{n}$ host nest using the following condition satisfied.

Set the iteration count value is $i=1$, Fitness score $i=i+1$.

Condition 2: Fitness evaluation

The fitness function casted off to examine the pluses of the distinctive in a category whose utility are must be non-negative, so it is generally portray from the unbiased task of the complication trim to get its maximum state. In a particular demand, the blueprint of the fitness function straight away impacts the rendering of CSA. For Quality of Service-aware service selection, the fitness function is expressed below.

Step 3: Find the fitness function for the generated input population of the cuckoo host or Qos parameters. Here the input Qos attributes are evaluated by using the CS algorithm.

The optimization problem (7) is defined as follows:

$$
\Phi(\tau)=\mu \mathrm{\imath} v\left(E_{\varnothing}\right)
$$

Where, $\min \left(E_{\varnothing}\right)$ is represents the minimization of QoS error function. The fitness function $\mathrm{F}(\mathrm{t})$ has to be minimized by arranging the services in an optimal way in the explore dominance of its boundary.

Step 4: Find the maximum and minimum fitness parameters of the first population values. From the performance analysis thus the output of the minimum fitness values are chosen as the best trained data solution.

Condition 3: Updating (new cuckoo generation)

To generate the new output solution using the levy flight search and find the fitness values. 
Step 5: Generally, the levy flight is the hunting route of an animal is fruitfully a directionless walk because the next move is based on both the present area and the change probability to the next solution. The path is depends on the probability so that it may be modeled mathematically.

A cuckoo without any order generates new outcomes by using the Levy function, and establishes the standard of solutions. The Levy function $\operatorname{Levy}(\lambda)$ in $(8)$ is represented as follows:

$$
X_{i}^{t+1}=X_{i}^{n}+\alpha \oplus \operatorname{Levy}(\lambda)
$$

Where, $\alpha$ is represents the duration length, where it has to be related to the scale of the muddle of engrossment. The outcome $\bigoplus$ indicates arrival-wise multiplications. The Cuckoo algorithm is assessed using the fitness function to decide the standard of the outcomes.

Step 6: Check probability

Discover the subdue roost framework construct on the probability $\left(\mathrm{P}_{\mathrm{a}}\right)$ and substitute the worst nests values by novel set of solutions. If the fitness function is minimized and satisfied the condition then the current solution is saved as an optimal solution. Otherwise, the earlier outcome is taken as the best outcome. Otherwise go to the step 3 still the condition is satisfied do the steps repeated.

Condition 4: Evaluate best trained data

Step 7: On the occasion of the step task is completed the CS algorithm is willing to provide the best trained dataset for the step response. The output is designated as specified by their inputs and the concurred output is recorded. In [30], at end of the process the best trained dataset value is analyzed. The flowchart of the proposed CS algorithm is presented in Fig. 2.

The best trained datasets are given as input of BN system to find a best Quality of Service -aware web service. Here the Bayesian network Classifier is one of algorithms to construct the system. The different steps of proposed BN model are expressed below.

The step procedure of BN system

Step 1: Evaluate the variables

In this section, the data are collected based on the QoS attributes. The input of best training dataset is evaluated with the help of CSA. The explanation of the training purpose is analyzed below.

Step 2: To Select variables for the final model.

Calculate correlative facts between each pair of correlatives base from (9),

$$
I\left(A_{I} ; A_{J}\right)=\sum_{i} \sum_{j} p\left(a_{I i}, a_{J j}\right) \log ^{p\left(a_{I i}, a_{J j}\right)} / p\left(a_{I i}\right) \times p\left(a_{J j}\right)
$$

Step 3: Calculate starting point between each pair of qualities.

Then select the smallest number of irregular $\mathbf{J}$ from the whole pool of $\mathrm{m}$ irregulars. It is written in (10) as,

$$
\sum_{j}^{J} I\left(\sigma, A_{j}\right) \geq F_{\sigma a} \sum_{m=1}^{m} I\left(\sigma, A_{k}\right)
$$

Where, $F_{\sigma a}$ is expressed as the uneven-assortment threshold

A worth of 1 specifies that the replica should include all the variables. Lesser values pointed out the minimal factual variables must be debarred. Grant the class node be the source of each picked variable.

Step 4: Select the connection in the middle of the preferred variables. Calculate the subject to correlative information between all the duos of variables, and subsequently grade the result.

\section{Step 5: Construct CPT}

CPT is deliberated for all the nodes in network structure. When node is positive then selected edge $=1$ or node is negative at that time selected edge $=0$. Then find the best optimal results of the web service. The proposed $\mathrm{BN}$ is performed with the CSA and the performance parameters are estimated and evaluated. The result of the proposed technique is outlined in following section. 


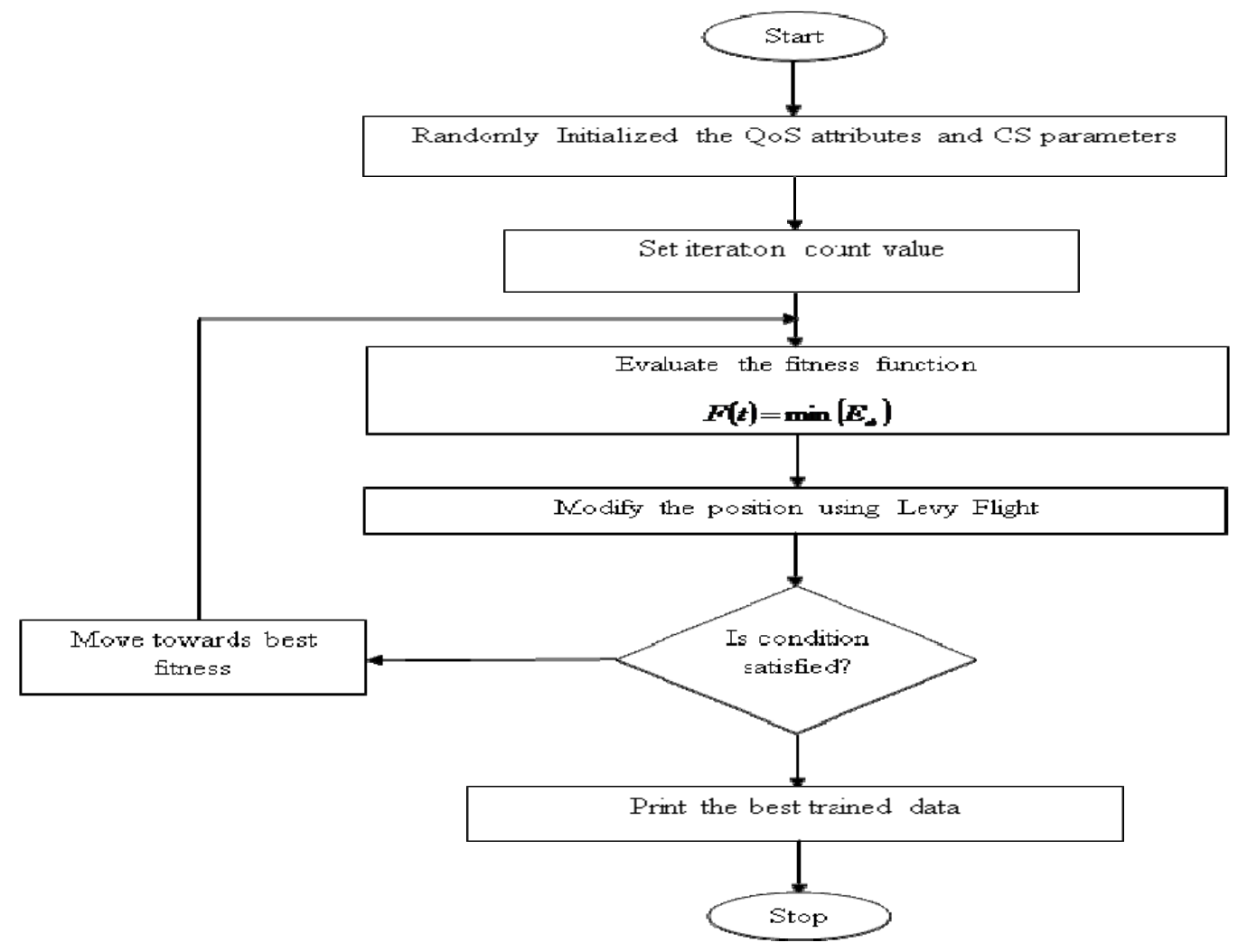

Fig. 2. The Flowchart of the Cuckoo search algorithm

\section{RESUlts AND Discussions}

The usefulness of our methodology has been verified on a wide set of arbitrarily produced instances. The proposed BN-CSA is applied to predict the optimal web service that satisfies service selection constraints and requirements. To show the performance of QoS aware web services using the proposed method, a test has been conducted. The experiment is carried out on a desktop PC with JAVA working platform. Here the proposed algorithm is performed to test the QoS attributes like reliability, availability, throughput, response time, and accuracy. The main objective of this experiment is to check whether the predicted result of the proposed Bayesian network with CSA is in line with different service levels as a consistent result to the service supports. Here we developed five works which are functionally different tests to know whether the prediction result of the proposed Bayesian network with CSA is in line with various service levels, the equivalent web services various service differentiation policies or not. In order to use BN to reason the optimal QoS-aware web service selection, First the system have to gather users different Quality of service requirements. Each and every service is call on many times with different Quality of Service requirements. At the end of each repetition the system come to have the service abilities to do the action using CSA and updates the corresponding B-Network. After that training of the BNetwork, the evaluation of the model could be used to forecast the web service selection of Quality of Service. The outcomes of the demonstrations showed that the proposed method can accurately estimate the Quality of Service selection, which is consistent to the various service levels, the service supports. Moreover the rendition of the proposed method is dis-similar with existing techniques namely, CSA, PSO and Fuzzy respectively.

\section{A. Performance analysis}

The experimental results showed that the Quality of Service optimization of services election can be achieved by using BN with CSA. Here, the best trained data is calculated by the development of CSA. The CSA is one of the current streamlining calculations in the class of nature based algorithm. To the best approach, there is a lack of real datasets that capture the QoS at different times and the corresponding quality class. Therefore, we can gather such data, and monitor the performance of real web services at different times. For example, they are designed for 100 different loading test cases for each of the monitored web services. In each of the test cases, delay in tine between them is calculated.. Moreover the delay and error response is tested and given better solution by adding the proposed method. The measurements of quality attributes are discussed. The response time (RT) of the web service selection is analyzed. Here the time spent between sending a request and receiving the last byte of the response is analyzed. This is measured in milliseconds. 


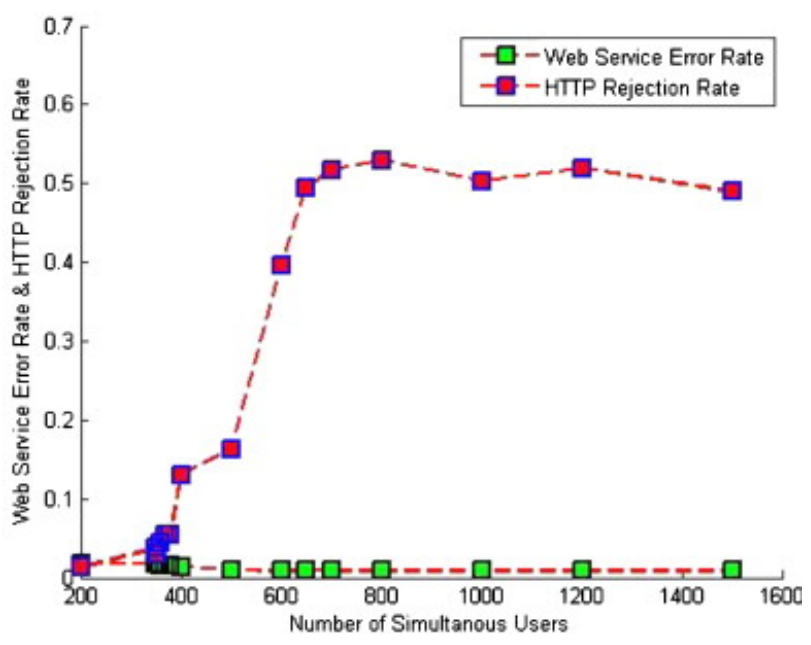

Fig. 3. Reliability of the QoS-aware web service

In Fig. 3 illustrates the reliability of web service with respect to the number of service users. The QoS attribute of reliability is described as the ratio of the number of valid responses to that of received ones. From the experimental result shows, the web service error is less and the HTTP rejection rate is high. Therefore the application of web services is quickly performed with the reduction of error. Hence, according to the results of web service rate and HTTP rejection rate is much effective by using the proposed method.

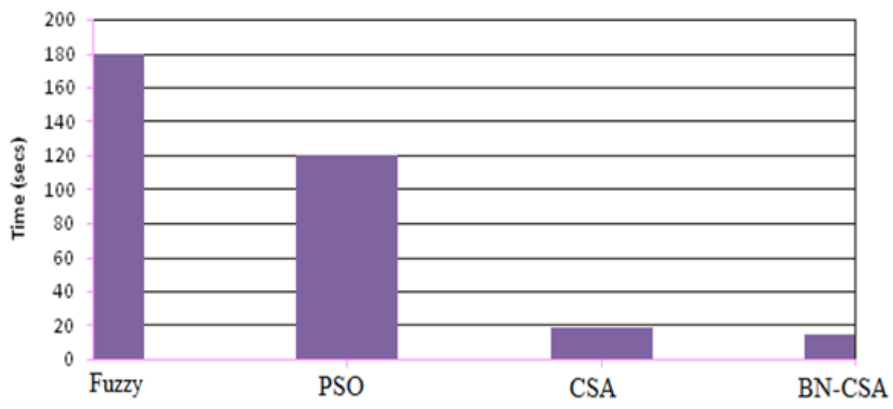

Fig. 4. Time complexity of the web service selection

In Fig. 4 analyzed the time complexity of the service selection with respect to time in sec. By using the proposed methodology, Web service is calculated in the time complexity. In the proposed work, the time complexity is below $15 \mathrm{sec}$. Here the performance of time complexity is compared with other techniques like fuzzy, PSO and CSA. The time complexity of the proposed method is short up to $15 \mathrm{sec}$. Similarly, the complexity of time is measured in other existing methods. The time complexity is too short. Hence, in line with the average computation time of the other approaches, the proposed method is able to find best services with shorter time complexity than other techniques. From those things, the proposed work achieves better outcomes as output in compared with many other techniques.

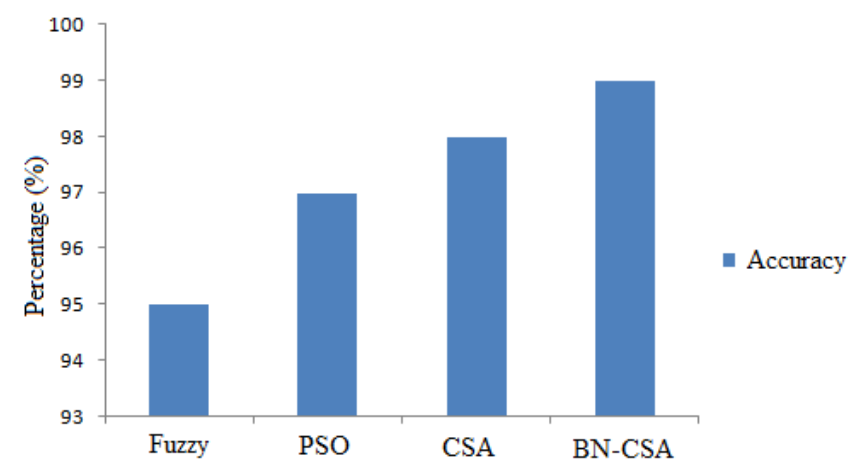

Fig. 5. Comparison of accuracy in QoS attributes 
In the proposed method, the performance of accuracy in percentage (\%) is analyzed. The performances are compared with the CSA, PSO and Fuzzy technique respectively. In proposed BN-CSA method, the accuracy of the service selection is increased up to $99 \%$. Similarly, the range of accuracy is measured in other existing methods namely CSA, PSO and Fuzzy are reduced up to $98 \%, 97 \%$ and $95 \%$ respectively. When compared to other techniques, one of the attribute in QoS accuracy is high in the proposed method. Then the experimental results of comparisons are illustrated in the Fig. 5. The problem is deals with minimization, it can be seen that comparatively BN-CSA has produced accurate results. The performance of proposed BN-CSA is investigated, whose results are superior to the CSA, PSO and Fuzzy system. The robustness of the proposed controller is demonstrated through some performance indices. It is clear from the Experimental evaluation, that the values of these system performances with the BN-CSA are minimum rate of error, faster response and high accuracy compared with those of CSA, PSO and fuzzy system respectively. This confirms that the reliability, availability, throughput, response time and accuracy of all attribute are greatly optimized by applying the proposed BN with CSA. From the overall performance analysis the optimization of Quality of Service - aware web service selection can be written as BN$\mathrm{CSA}>\mathrm{CSA}>\mathrm{PSO}>$ Fuzzy respectively.

\section{CONCLUSION}

In the paper, a BN-CSA is proposed to optimize the selection of QoS-aware web services. The proposed work is effective in action to predict Quality of service requirements of user in various combinations. In particular, to the provider with multiple service levels which are different in each service levels. QoS model for web services is adopted based on widely used QoS criteria, namely, execution time, reliability, density and accuracy of success. Initially, the CSA is performed to find the best trained dataset values. After that the training data is given as input of $\mathrm{BN}$. The proposed $\mathrm{BN}$ is used to minimize the error function and optimize the selection of 'QoS aware web service'. The performance of proposed method is implemented in JAVA working platform and compared with the existing techniques namely CSA, PSO and Fuzzy. The output function of the proposed technique gives good accuracy $(99 \%)$ of the operation. The experimental results on the proposed datasets demonstrate that this method has better results compared with other techniques, and shows that the proposed method is efficient to optimize the Selection of Quality of Service - aware Web Service.

\section{REFERENCES}

[1] Li, M., D. Zhu, T. Deng, H. Sun, H. Guo and X. Liu, GOS: a global optimal selection strategies for QoS-aware web services composition. Service Oriented Computing and Applications, DOI: 10.1007/s11761-013-0133-7, Vol. 7, pp.181-197, 2013.

[2] Guo, H., F. Tao, L. Zhang, S. Su and Nan Si, Correlation-aware web services composition and QoS computation model in virtual enterprise. The International Journal of Advanced Manufacturing Technology, DOI: 10.1007/s00170-010-2648-9, Vol. 51, pp. 5-8, 2010.

[3] Arulanand, N., and P. M. Ananth, Optimized Weight Vector for QoS Aware Web Service Selection Algorithm Using Particle Swarm Optimization. World Academy of Science, Engineering and Technology, Vol. 9, pp. 993-998, 2015.

[4] Zou, G., Y. Xiang, Y. Gan, D. Wang and Z. Liu, An agent-based web service selection and ranking framework with QoS.In proceedings of IEEE conferences on Computer Science and Information Technology, Aug. 8-11, DOI: 10.1109/ICCSIT.2009.5234607, pp. 37-42, 2009.

[5] Hadad, E. H., M. Manouvrier and M. Rukoz, TQoS: Transactional and QoS-aware selection algorithm for automatic Web service composition. IEEE Transactions on Services Computing, DOI: 0.1109/TSC.2010.5, Vol. 3, pp. 73-85, 2010.

[6] Wang, P., QoS-aware web services selection with intuitionistic fuzzy set under consumer's vague perception. Expert Systems with Applications, DOI: 0.1016/j.eswa.2008.05.007, Vol. 36, pp. 4460-4466, 2009.

[7] Zeng, L., B. Benatallah, A. H. H. Ngu, M. Dumas, J. Kalagnanam and H. Chang, Qos-aware middleware for web services composition. IEEE Transactions on software engineering, DOI: 10.1109/TSE.2004.11, Vol. 30, pp. 311-327, 2004.

[8] Qi, L., W. Dou, X. Zhang and J. Chen, A QoS-aware composition method supporting cross-platform service invocation in cloud environment. Journal of Computer and System Sciences, DOI: 10.1016/j.jcss.2011.12.016, Vol. 78, pp. 1316-1329, 2012.

[9] Yang, Y., H. Ma, and M. Zhang, A genetic programming approach to distributed qos-aware web service composition.In proceedings of IEEE conference on Evolutionary Computation (CEC), July. 6-11, DOI: 10.1109/CEC.2014.6900416, pp: 1840-1846, 2014.

[10] Zhang, C., S. S, and J. Chen, DiGA: Population diversity handling genetic algorithm for QoS-aware web services selection. Computer Communications, DOI: 10.1016/j.comcom.2006.11.002, Vol. 30, pp. 1082-1090, 2007.

[11] Canfora, G., M. Di Penta, R. Esposito and M. L. Villani, QoS-aware replanning of composite web services.In proceedings of IEEE confereces on Web Services, July. 11-15, DOI: 10.1109/ICWS.2005.96, pp: 121-129, 2005.

[12] Canfora, G., M. D. Penta, R. Esposito and M. L. Villani, A framework for QoS-aware binding and re-binding of composite web services. Journal of Systems and Software, DOI: 10.1016/j.jss.2007.12.792, Vol. 81, pp. 1754-1769, 2008.

[13] Xiao Y. Y. and A. Wang, Genetic Algorithm Based Bayesian Network for Customers' Behavior Analysis. In proceedings of IEEE conference on Intelligent Information Hiding and Multimedia Signal Processing, Oct. 15-17, DOI: 10.1109/IIHMSP.2010.104, pp. 406-409, 2010.

[14] Mohammed, M., M. A. Chikh and H. Fethallah, Qos-aware web service selection based on harmony search. In proceedings of IEEE conferences on Concepts and Tools for knowledge Management (ISKO-Maghreb), Nov. 9-10, DOI: 10.1109/ISKOMaghreb.2014.7033465, pp. 1-6, 2014.

[15] Huang, J., G. Liu, Q. Duan and Y. Yan, Qos-aware service composition for converged network-cloud service provisioning. In proceedings of international conference on Services Computing (SCC), June 27 - July 2, DOI: 10.1109/SCC.2014.18, pp. 67-74, 2014.

[16] Ma, Y. and C. Zhang, Quick convergence of genetic algorithm for QoS-driven web service selection. Computer Networks, DOI: 10.1016/j.comnet.2007.12.003, Vol. 52, pp. 1093-1104, 2008.

[17] Mousa, A. and J. Bentahar, An Efficient QoS-aware Web Services Selection Using Social Spider Algorithm. Procedia Computer Science, DOI: 10.1016/j.procs.2016.08.027, Vol. 94pp. 176-182, 2016. 
[18] Kobti, Z., and W. Zhiyang, An adaptive approach for QoS-aware web service composition using cultural algorithms. In Proceedings of the 20th Australian joint conference on Advances in artificial intelligence, Dec. 2-6, DOI: 10.1007/978-3-540-76928-6_16, pp. 140$149,2007$.

[19] Rajeswari, M., G. Sambasivam, N. Balaji, MS SaleemBasha, T. Vengattaraman and P. Dhavachelvan, Appraisal and analysis on various web service composition approaches based on QoS factors. Journal of King Saud University-Computer and Information Sciences, DOI: 10.1016/j.jksuci.2013.08.003, Vol. 26, pp. 143-152, 2014.

[20] Rodriguez-Mier, P., M. Mucientes and Manuel Lama, Hybrid optimization algorithm for large-scale QoS-aware service composition.IEEE transactions on services computing, DOI: 10.1109/TSC.2015.2480396, Vol. 10, pp. 547 - 559, 2015.

[21] Wagner, F., F. Ishikawa and S. Honiden, Robust service compositions with functional and location diversity.IEEE Transactions on Services Computing,. DOI: 10.1109/TSC.2013.229579, Vol. 9, pp. 277-290, 2016.

[22] Bekkouche, A., S. M. Benslimane, M. Huchard, C. Tibermacine, F. Hadjila and M. Merzoug, QoS-aware optimal and automated semantic web service composition with user's constraints. Service Oriented Computing and Applications,. DOI: 10.1007/s11761-0170205-1, Vol. 11, pp. 183-201, 2017.

[23] Wu, Q., F. Ishikawa, Q. Zhu and D. Shin, QoS-Aware Multigranularity Service Composition: Modeling and Optimization. IEEE Transactions on Systems, Man, and Cybernetics: Systems, DOI: 10.1109/TSMC.2015.2503384, Vol. 46, pp. 1565-1577, 2016.

[24] Zhou, J., and X. Yao, A hybrid artificial bee colony algorithm for optimal selection of QoS-based cloud manufacturing service composition. The International Journal of Advanced Manufacturing Technology, DOI: 10.1007/s00170-016-9034-1, Vol. 88, pp. 3371-3387, 2017.

[25] Z. Ding, Y. Sun, J. Liu, M. Pan and J. Liu, A genetic algorithm based approach to transactional and QoS-aware service selection. Enterprise Information Systems, DOI: 10.1080/17517575.2015.1048832, Vol. 11, pp. 339-358, 2017.

[26] Ding, S., Xia, C., Wang, C., Wu, D. and Zhang, Y., Multi-objective optimization based ranking prediction for cloud service recommendation. Decision Support Systems, DOI: 0.1016/j.dss.2017.06.005, Vol. 101, pp. 106-114, 2017.

[27] Mao, C., J. Chen, D. Towey, J. Chen and X. Xie, Search-based QoS ranking prediction for web services in cloud environments. Future Generation Computer Systems, DOI: 10.1016/j.future.2015.01.008, Vol. 50, pp. 111-126, 2015.

[28] Wu, G., J. Wei, X. Qiao and L. Li, Bayesian network based Qos assessment model for web services. In proceedings of IEEE conference on Services Computing, July 9-13, DOI: 10.1109/SCC.2007.1, pp. 1-8, 2007.

[29] Wang, W., Q. Sun, X. Zhao and F. Yang, An improved particle swarm optimization algorithm for QoS-aware web service selection in service oriented communication. International Journal of Computational Intelligence Systems, DOI: 10.1080/18756891.2010.9727750, Vol. 3, pp. 18-30, 2010.

[30] Abd, E. S. M. and E.S. Ali, Optimal Power System Stabilizers design via Cuckoo Search algorithm. Electrical Power and Energy Systems, DOI: 10.1016/j.ijepes.2015.08.018, Vol. 75, pp, 99-107, 2016.

\section{AUTHORS PROFILE}

Saravanan chandrasekaran is pursuing the Ph.D degree in department of Computer Science and Engineering in Annamalai University. He is currently working as a Assistant Professor in the department of Information Technology, Krishnasamy college of Engineering and Technology, Anna University, India. His research area includes Web Services, Cloud Computing.

Vijay Bhanu Srinivasan received his Ph.D. degree in Computer Science and Engineering from Anna University, Coimbatore, India. He is currently working as a Assistant Professor in Department of Computer Science and Engineering at Annamalai University, India. His research interests include Computer Networks, Web Services, Cloud Computing, Software Engineering and Software Testing.

Latha Parthiban received her B.E in 1994, M.S in 1997and Ph.D in 2010. She has 20 years of teaching in engineering colleges in Chennai including 4 year at Pondicherry University Community College. She has published 76 international/national journals, 6 books with international publications and published papers in 40 international/national conferences. She is the Editor in chief of International Journal of Human Machine Interface of Artificial Intelligence Association of India. She has reviewed text books for leading publishers like oxford. She has also reviewed papers for many international journals including IEEE, Elsevier, Springer link etc. 